\title{
Recalcitrant Hypocalcemia in Roux-en-Y Gastric Bypass Patients \\ After Parathyroidectomy: Successful Management With
}

\author{
Gastrostomy Tube Placement
}

Danielle Hayes, $\mathrm{MD}^{1 *}$; Lancy Tan, MD; Andrew Feczko, MD'; Joseph Sniezek, MD²

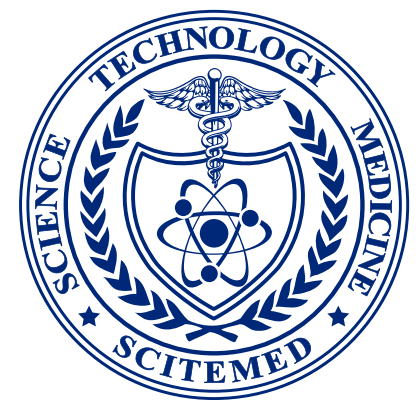

'Department of Surgery, Swedish Medical Center, Seattle, Washington, USA

${ }^{2}$ Department of Head and Neck Surgery, Swedish Medical Center, Seattle, Washington, USA

\begin{abstract}
Thyroid and parathyroid surgery in patients with history of Roux-en-Y gastric bypass (RYGB) is associated with increased risk of postoperative hypocalcemia. We present a case report of a patient with history of RYGB who underwent thyroidectomy and parathyroidectomy with postoperative development of severe recalcitrant hypocalcemia. Hypocalcemia was managed conservatively initially but subsequently required gastrostomy tube placement with successful resolution of hypocalcemia.
\end{abstract}

\section{Introduction}

Since 2011, bariatric procedures in the United States have increased by $44 \%$ [1]. This increase in bariatric procedures has resulted in new challenges in perioperative management due to the alterations in gut physiology and absorption. In Roux-en-Y gastric bypass (RYGB), oral nutrition bypasses the stomach to the proximal small intestine, resulting in decreased calcium and vitamin D absorption and disruption of intrinsic endocrine function [2]. In most RYGB patients, normocalcemia is maintained with a compensatory secondary hyperparathyroidism. However, thyroid and parathyroid surgery can severely impact this endocrine balance. We present a case study of a patient with history of RYGB who presented with recalcitrant hypocalcemia after thyroidectomy and parathyroidectomy with novel management using gastrostomy tube.

\section{Case Report}

A 40-year-old female with history of RYGB was found on postoperative follow up to have symptomatic hypercalcemia $(10.5 \mathrm{mg} / \mathrm{dL})$ with elevated parathyroid hormone (PTH) $(272 \mathrm{pg} / \mathrm{mL})$ and a $3 \mathrm{~cm}$ hypoechoic lesion consistent with parathyroid adenoma. Multiple bilateral thyroid gland nodules were also identified without laboratory evidence of hyperthyroidism. Parathyroidectomy was recommended and total thyroidectomy was offered due to her desire to avoid ongoing surveillance for thyroid nodules.

She underwent an uncomplicated parathyroidectomy and total thyroidectomy. A $3 \mathrm{~cm}$ right inferior parathyroid adenoma was identified and removed with an appropriate intraoperative decrease in PTH level from $328 \mathrm{pg} / \mathrm{mL}$ to $47 \mathrm{pg} / \mathrm{mL}$ after 15 minutes. The right and left superior parathyroid glands were preserved in situ. The left inferior parathyroid gland was re- implanted into the ipsilateral sternocleidomastoid muscle due to concerns for its vascular supply. Pathology confirmed hyperplastic parathyroid adenoma.

Postoperatively, the patient was started on calcium and vitamin $D$ supplementation. She was discharged on postoperative day 1 with calcium $8.7 \mathrm{mg} / \mathrm{dL}$ and PTH 7pg/mL. She subsequently returned on postoperative day 2 with perioral and distal extremity paraesthesias. Repeat serum calcium was $7.2 \mathrm{mg} / \mathrm{dL}$ and ionized calcium $1.02 \mathrm{mmol} / \mathrm{L}$. The patient was admitted, and calcium was administered aggressively both enterally and intravenously (16g intravenous calcium and $8 \mathrm{~g}$ enteral calcium daily). She was eventually started on a continuous intravenous (IV) calcium gluconate infusion at rate of $1.075 \mathrm{~g} / \mathrm{hr}$ to maintain normocalcemia even though she maintained normal PTH levels beginning on post-operative day 4 . Multiple attempts to wean the patient from the calcium infusion were unsuccessful despite the normal levels of PTH.

On hospital day 10, the patient underwent laparoscopic gastrostomy tube placement. She was started on enteral supplements through the gastrostomy tube the following day. She was transitioned off calcium infusion on post-procedure day 5 (hospital day 16), and discharged on hospital day 18. After discharge, she returned for daily calcium infusions for four days following discharge and labs were drawn daily for one week, then weekly for three weeks. She was seen in the follow-up clinic one week after discharge and weekly in bariatric and endocrine clinic for three weeks. Calcium citrate was started orally two weeks post discharge and calcium carbonate via gastrostomy tube was discontinued. The gastrostomy tube was removed at week 3 . She has remained asymptomatic at three-month follow-up.

\section{Discussion}

This case demonstrates novel management of rare, but serious, refractory hypocalcemia in patients undergoing parathyroid and thyroid surgery following RYGB. Initial management involves aggressive calcium repletion which often necessitates prolonged hospital stays [3-6]. In cases where conservative management fails, reversal of RYGB has been reported in the literature $[7,8]$. To our knowledge, this is the first reported instance where hypocalcemia refractory to conservative therapy was managed surgically through gastrostomy tube only, facilitating access to the bypassed RYGB anatomy without a complete reversal of RYGB.

Calcium and vitamin $D$ malabsorption is a known result of RYGB. Active calcium transport occurs in the duodenum which is bypassed, disrupting absorption of enteric sources of calcium. Passive calcium absorption is facilitated by an acidic environment which is also impaired by the bypassed stomach. Vitamin D absorption in the proximal jejunum is also altered. Most RYGB patients, however, maintain normocalcemia due to compensatory secondary hyperparathyroidism where physiologic normocalcemia is maintained to the detriment of the bony calcium pool [9]. Transient disruption of increased parathyroid function following a thyroidectomy or removal of a hyperfunctioning parathyroid adenoma leads to increased calcium deposition into bone, leading to a decrease in serum calcium which can be symptomatic depending on its extent. This so called hungry bone syndrome (HBS) is well described following parathyroidectomy and usually can be managed with aggressive oral and IV replacement of calcium and vitamin D [10]. 
The development of HBS following thyroid or parathyroid surgery in patients with RYGB is complicated by the limited absorption of calcium, magnesium and vitamin $D$ that occurs with the reconstructed anatomy [4]. Symptomatic and persistent hypocalcemia in these patients has been described in both the bariatric and endocrine surgery literature and typically responds to aggressive replacement of calcium both enterally and parenterally until either parathyroid function improves, and/or the bony calcium pool is reconstituted [10]. Reversal of the bypass anatomy has been described for refractory HBS to improve enteral absorption of calcium and move patients beyond needing frequent infusions of IV calcium $[11,12]$. Campos et al. reported one case of hypocalcemia secondary to hypoparathyroidism after thyroidectomy with successful management via RYGB reversal. Gastrostomy tube was placed prior to confirm that reversal would be effective, however, they did not report the efficacy of gastrostomy tube placement itself in resolution of hypocalcemia [11]

Our approach to this patient with persistent HBS was to utilize the normal absorptive anatomy of the duodenum and proximal jejunum, functionally reversing the RYGB with laparoscopic placement of a gastrostomy tube into the gastric remnant. This avoided the complications of a larger surgery and the minimally invasive technique was well tolerated. A gastrostomy tube allowed for the transition to an enteral regimen of calcium, vitamin D and magnesium replacement. The patient was subsequently weaned from continuous IV calcium replacement to an outpatient regiment of IV infusions and then to only enteric calcium.

We believe we are the first to report this novel management strategy for a patient with HBS following head and neck endocrine surgery. Most patients will not require this intervention, however, earlier consideration of this surgical approach may expedite recovery of normocalcemia and minimize the need for ongoing hospitalization to monitor serum calcium levels and provide IV replacement. Additionally, the placement of a gastrostomy tube allows for the provision of a small amount of caloric intake (tube feeds) through the gastric remnant and duodenum to enhance mucosal calcium absorption without obliviating the restrictive and malabsorptive effects of the remaining RYGB.

\section{Conclusion}

It is important for surgeons planning endocrine procedures to be cognizant of sequelae of bariatric surgery. Patients who have undergone RYGB need to be monitored closely when undergoing thyroid and parathyroid surgery. Early gastrostomy tube placement for enhanced enteral absorption of calcium and vitamin D may be an option for patients with recalcitrant hypocalcemia resistant to intravenous and enteral replacement alone.

\section{Article Information}

* Correspondence: Danielle Hayes, MD

Department of Surgery, Swedish Medical Center, First Hill Campus, 747 Broadway, Heath Tenth, Seattle, WA, 98122, USA.

E-mail: danielle.hayes@swedish.org

Received: Aug. 29, 2018; Accepted: Sep. 24, 2018; Published: Oct. 13, 2018

DOI: $10.24983 /$ scitemed.aohns.2018.00086
Copyright (c) 2018 The Author (s). This is an open-access article distributed under the terms of the Creative Commons Attribution 4.0 International License (CC-BY).

\section{Funding: None}

\section{Conflict of Interest: None}

\section{Keywords}

Gastrostomy tube; hypocalcemia; parathyroidectomy; Roux-en-Y gastric bypass; thyroidectomy.

\section{References}

1. American Society for Metabolic and Bariatric Surgery. Estimate of bariatric surgery numbers, 2011-2017. Available at: https://asmbs.org/ resources/estimate-of-bariatric-surgery-numbers. Accessed August 29, 2018.

2. Schafer AL, Weaver CM, Black DM, et al. Intestinal calcium absorption decreases dramatically after gastric bypass surgery despite optimization of vitamin D status. J Bone Miner Res 2015;30(8):1377-1385.

3. Pietras SM, Holick MF. Refractory hypocalcemia following near- total thyroidectomy in a patient with a prior Roux-en-Y gastric bypass. Obes Surg 2009;19(4):524-526.

4. McKenzie TJ, Chen Y, Hodin RA, et al. Recalcitrant hypocalcemia after thyroidectomy in patients with previous Roux-en-Y gastric bypass. Surgery 2013;154(6):1300-1306.

5. Allo Miguel G, García Fernández E, Martínez Díaz-Guerra G, et al. Recalcitrant hypocalcemia in a patient with post-thyroidectomy hypoparathyroidism and Roux-en-Y gastric bypass. Obes Res Clin Pract 2016;10(3):344-347.

6. Chereau N, Vuillermet C, Tilly $C$, et al. Hypocalcemia after thyroidectomy in patients with a history of bariatric surgery. Surg Obes Relat Dis 2017;13(3):484-490.

7. Campos GM, Ziemelis M, Paparodis R, Ahmed M, Davis DB. Laparoscopic reversal of Roux-en-Y gastric bypass: technique and utility for treatment of endocrine complications. Surg Obes Relat Dis 2014;10(1):36-43.

8. Davis DB, Khoraki J, Ziemelis M, Sirinvaravong S, Han JY, Campos GM. Roux en $Y$ gastric bypass hypoglycemia resolves with gastric feeding or reversal: confirming a non-pancreatic etiology. Mol Metab 2018;9:15-27.

9. Shah M, Sharma A, Wermers RA, Kennel KA, Kellogg TA, Mundi MS. Hypocalcemia after bariatric surgery: prevalence and associated risk factors. Obes Surg 2017;27(11):2905-2011.

10. Witteveen JE, van Thiel S, Romijn JA, Hamdy NA. Hungry bone syndrome: still a challenge in the post-operative management of primary hyperparathyroidism: a systematic review of the literature. Eur J Endocrinol. 2013; 168(3):R45-53.

11. Palal B, Sinsakul M, Reutrakul S. Life-threatening hypocalcemia following subtotal parathyroidectomy in a patient with renal failure and previous Roux-en-Y gastric bypass surgery. Case Rep Endocrinol 2011;2011:370583.

12. Chen $Y$, Lubitz CC, Shikora SA, et al. Primary hyperparathyroidism after Roux-en-Y gastric bypass. Obes Surg 2015;25(4);700-704. 